\title{
Spatially-resolved imaging of stripped-envelope supernova environments
}

\author{
Schuyler D. Van Dyk \\ Caltech, 100-22, Pasadena, CA 91125 USA \\ email: vandyk@ipac.caltech.edu
}

\begin{abstract}
Stripped-envelope supernovae (SNe), i.e., those of Type Ib, Ic, and IIb, arise from massive progenitor stars which have had most or all of their outer hydrogen-rich layers removed before explosion by some process, either through a strong stellar wind or through binary mass transfer. The connection between some long-duration gamma-ray bursts (GRBs) and broadlined Type Ic SNe makes a broader discussion of stripped-envelope SNe and their environments particularly relevant. If the SN progenitor itself cannot be directly identified, it is possible that examination of its immediate environment can provide some insight into the nature of the progenitor. It is also possible that revisiting the SN site sufficiently late enough after explosion could reveal the presence of a binary companion. I will present high-spatial-resolution observations obtained with the Hubble Space Telescope of the sites and environments of stripped-envelope supernovae, and I will discuss the implications of the resulting analysis. I will include here, e.g., the environments of the recent SN 2011dh, SN 2012au, SN 2013df, SN 2013dk, and iPTF13bvn.
\end{abstract}

Keywords. supernovae: individual (SN 1994I, SN 2008ax, SN 2011dh, iPTF13bvn); stars: evolution; binaries (including multiple): close; stars: Wolf-Rayet; galaxies: individual (NGC 4490, NGC 5194, NGC 5806); galaxies: stellar content

\section{Summary}

Stripped-envelope SN (SESN) environments are, generally, either crowded, dusty (e.g., the SN Ic 2003jg environment, Eldridge et al. 2013; Drout et al. 2011 found that $E[B-$ $V] \gtrsim 0.4$ mag for their SN sample), or both (e.g., the SN Ic 2013dk environment; EliasRosa et al. 2013). One means of constraining progenitor initial masses for SESNe is by analyzing stellar populations in the immediate environment (e.g., Murphy et al. 2011; Williams et al. 2014), resolved by the Hubble Space Telescope (HST). The SN IIb 2011dh progenitor was directly identified to be a yellow supergiant in pre-SN HST multi-band images from 2005 of the host galaxy, NGC 5194 (Van Dyk et al. 2011; Maund et al. 2011). Van Dyk et al. (2013) subsequently demonstrated with late-time HST images that this star had vanished. A UV-bright source was detected with HST at the SN position in 2014; Folatelli et al. (2014) interpreted this detection in the context of their interacting binary models (although see Maund et al. 2015). Van Dyk et al. (2015) searched for the binary companion to the progenitor of SN Ic 1994I (also in NGC 5194), and the non-detection constrains the companion to a hot dwarf with $M \lesssim 10 M_{\odot}$; they further interpreted this result in light of binary population synthesis simulations. Crockett et al. (2008) reasoned that the "compact" progenitor of SN IIb 2008ax in NGC 4490 was likely a single Wolf-Rayet (WR) star. Folatelli et al. (2015) reanalyzed the pre-SN HST progenitor photometry, also considering the non-detection of a binary companion in very late-time, multi-band HST imaging, and once again interpreted these results using their binary evolution models. Our independent analysis (SVD, O.D. Fox, N. Smith, S.E. deMink, et al., in prep.) results in a somewhat more luminous progenitor for SN 2008ax, not necessarily requiring it to be in a binary. Cao et al. (2013) nominally made the first 
progenitor detection for a SN Ib, iPTF13bvn in NGC 5806. We (SVD, G. Folatelli, H. Kuncarayakti, et al., in prep.) are currently analyzing late-time, multi-band HST imaging of this SN site to determine whether this was indeed the case. Support was provided by NASA through a grant from STScI, which is operated by AURA, Inc., under NASA contract NAS 5-26555.

\section{References}

Cao, Y., Kasliwal, M. M., Arcavi, I., Horesh, A., Hancock, P., Valenti, S., Cenko, S. B., Kulkarni, S. R., Gal-Yam, A., Gorbikov, E., Ofek, E. O., Sand, D., et al. 2013, ApJL, 775, L7

Crockett, R. M., Eldridge, J. J., Smartt, S. J., Pastorello, A., Gal-Yam, A., Fox, D. B., Leonard, D. C., Kasliwal, M. M., Mattila, S., Maund, J. R., et al. 2008, MNRAS, 391, L5

Drout, M. R., Soderberg, A. M., Gal-Yam, A., Cenko, S. B., Fox, D. B., Leonard, D. C., Sand, D. J., Moon, D.-S., Arcavi, I., \& Green, Y. 2013, ApJ, 741, 97

Elias-Rosa, N., Pastorello, A., Maund, J. R., Takáts, K., Fraser, M., Smartt, S. J., Benetti, S., Pignata, G., Sand, D., \& Valenti, S. 2013, MNRAS, 436, L109

Eldridge, J. J., Fraser, M., Smartt, S. J., Maund, J. R., \& Crockett, R. M. 2013, MNRAS, 436, 774

Folatelli, G., Bersten, M. C., Benvenuto, O. G., Van Dyk, S. D., Kuncarayakti, H., Maeda, K., Nozawa, T., Nomoto, K., Hamuy, M., \& Quimby, R. M. 2014, ApJL, 793, L22

Folatelli, G., Bersten, M. C., Kuncarayakti, H., Benvenuto, O. G., Maeda, K., \& Nomoto, K. 2015, ApJ, 811, 147

Maund, J. R., Fraser, M., Ergon, M., Pastorello, A., Smartt, S. J., Sollerman, J., Benetti, S., Botticella, M.-T., Bufano, F., Danziger, I. J., Kotak, R., et al. 2011, ApJL, 739, L37

Maund, J. R., Arcavi, I., Ergon, M., Eldridge, J. J., Georgy, C., Cenko, S. B., Horesh, A., Izzard, R. G., \& Stancliffe, R. J. 2015, MNRAS, 454, 2580

Murphy, J. W., Jennings, Z. G., Williams, B., Dalcanton, J. J., \& Dolphin, A. E. 2011, ApJL, $742, \mathrm{~L} 4$

Van Dyk, S. D., Li, W., Cenko, S. B., Kasliwal, M. M., Horesh, A., Ofek, E., Kraus, A. L., Silverman, J. M., Arcavi, I., Filippenko, A. V., Gal-Yam, A., et al. 2011, ApJL, 741, L28

Van Dyk, S. D., Zheng, W., Clubb, K. I., Filippenko, A. V., Cenko, S. B., Smith, N., Fox, O. D., Kelly, P. L., Shivvers, I., \& Ganeshalingam, M. 2013, ApJL, 772, L32

Van Dyk, S. D., de Mink, S. E., \& Zapartas, E. 2015, ApJ, submitted

Williams, B. F., Peterson, S., Murphy, J., Gilbert, K., Dalcanton, J. J., Dolphin, A. E., \& Jennings, Z. G. 2015, ApJ, 791, 105 